\title{
Differential cellular uptake and metabolism of curcuminoids in monocytes/macrophages: regulatory effects on lipid accumulation
}

\author{
Kiyotaka Nakagawa ${ }^{1,2 *}$, Jean-Marc Zingg $^{1} \dagger$, Sharon H. Kim ${ }^{1}$, Michael J. Thomas ${ }^{1}$, \\ Gregory G. Dolnikowski ${ }^{3}$, Angelo Azzi ${ }^{1}$, Teruo Miyazawa ${ }^{2}$ and Mohsen Meydani ${ }^{1}$ \\ ${ }^{1}$ Vascular Biology Laboratory, Jean Mayer USDA-Human Nutrition Research Center on Aging, Tufts University, Boston, \\ MA 02111 , USA \\ ${ }^{2}$ Food and Biodynamic Chemistry Laboratory, Graduate School of Agricultural Science, Tohoku University, \\ Sendai 981-8555, Japan \\ ${ }^{3}$ Mass Spectrometry Laboratory, Jean Mayer USDA-Human Nutrition Research Center on Aging, Tufts University, Boston, \\ MA, 02111, USA
}

(Submitted 6 December 2013 - Final revision received 17 January 2014 - Accepted 5 February 2014 - First published online 11 April 2014)

\section{Abstract}

We have previously shown that curcumin (CUR) may increase lipid accumulation in cultured human acute monocytic leukaemia cell line THP-1 monocytes/macrophages, but that tetrahydrocurcumin (THC), an in vivo metabolite of CUR, has no such effect. In the present study, we hypothesised that the different cellular uptake and/or metabolism of CUR and THC might be a possible explanation for the previously observed differences in their effects on lipid accumulation in THP-1 monocytes/macrophages. Chromatography with tandem MS revealed that CUR was readily taken up by THP-1 monocytes/macrophages and slowly metabolised to hexahydrocurcumin sulphate. By contrast, the uptake of THC was low. In parallel with CUR uptake, increased lipid uptake was observed in THP-1 macrophages but not with the uptake of THC or another CUR metabolite and structurally related compounds. From these results, it is possible to deduce that CUR and THC are taken up and metabolised differently in THP-1 cells, which determine their biological activity. The remarkable differential cellular uptake of CUR, relative to THC and other similar molecules, may imply that the CUR uptake into cells may occur via a transporter.

Key words: Curcuminoids: Lipids: Macrophages: Metabolism: Monocytes

Curcumin (CUR), also known as diferuloylmethane (IUPAC name (1E,6E)-1,7-bis(4-hydroxy-3-methoxyphenyl)-1,6-heptadiene-3,5-dione), is obtained from the rhizome of turmeric (Curcuma longa L.) and is present in dried turmeric powder at a concentration of $2-5 \%{ }^{(1)}$. CUR has been shown to have several physiological activities ${ }^{(2-5)}$, and we have previously shown that CUR may increase lipid accumulation in cultured human acute momocytic leukaemia cell line THP-1 monocytes/macrophages via up-regulation of the expression of lipid transport genes (fatty acid transporter CD36 (cluster of differentiation 36)/FAT) and fatty acid-binding protein-4 $(F A B P-4)^{(6)}$. Increased expression of CD36 in THP-1 macrophages induced by CUR has also been reported by others ${ }^{(7,8)}$. CUR-induced accumulation of lipids in macrophages may be part of a mechanism aimed at the removal of lipids from the bloodstream. Because tetrahydrocurcumin (THC), a metabolite resulting from CUR reduction, has no effect on lipid accumulation in vitro ${ }^{(6)}$, the presence of different cellular uptake and/or metabolism of CUR and THC may explain their differential effects on lipid accumulation in THP-1 monocytes/macrophages.

It is well known that upon absorption through intestinal epithelial cells in animals, most of the CUR is conjugated mainly to glucuronide, forming curcumin glucuronide (CURG $^{(9-11)}$. THC has been detected in the blood plasma of mice injected intraperitoneally with $\mathrm{CUR}^{(12)}$. Unmetabolised CUR is found in blood at extremely low concentrations ${ }^{(9,11)}$. On the other hand, little is known about the cellular uptake and metabolism of CUR in cultured cells ${ }^{(11,13-16)}$ other than those in hepatic and intestinal cells.

In the present study, using monocyte/macrophage cell lines and HPLC-tandem MS (MS/MS) techniques, we investigated the cellular uptake and metabolism of CUR and other related

Abbreviations: CUR, curcumin; CURG, curcumin glucuronide; DMC, demethoxycurcumin; FL, fluorescence; HHC, hexahydrocurcumin; HHCS, hexahydrocurcumin sulphate; MRM, multiple-reaction monitoring; PMA, phorbol 12-myristate 13-acetate; THC, tetrahydrocurcumin.

*Corresponding author: K. Nakagawa, fax +81 22717 8905, email nkgw@m.tohoku.ac.jp

†These authors contributed equally to the present study. 
molecules (e.g. THC and CURG) to gain insights into their differential effects on lipid accumulation in THP-1 macrophages in an in vitro cell-culture system ${ }^{(6)}$.

\section{Materials and methods}

\section{Cell culture}

The human acute monocytic leukaemia cell line THP-1 (ATCC TIB-202) was cultured in RPMI (Roswell Park Memorial Institute medium) 1640 medium (containing 2 mM-L-glutamine, $1 \mathrm{~mm}$-sodium pyruvate and $4.5 \mathrm{~g} / 1$ glucose) supplemented with $10 \%$ fetal calf serum, $100 \mathrm{U} / \mathrm{ml}$ penicillin and $100 \mu \mathrm{g} / \mathrm{ml}$ streptomycin at $37^{\circ} \mathrm{C}$ in $5 \% \mathrm{CO}_{2}-95 \%$ air atmosphere in a humidified incubator.

\section{Treatment}

CUR (purity $\geq 98.5 \%$; Enzo Life Sciences), THC (kindly provided by V. Badamov, Sbinsa Corporation) and CURG (Toronto Research Chemicals) were each dispersed in ethanol at a concentration of $20 \mathrm{~mm}$. THP-1 monocytes were seeded at $2 \times 10^{6}$ cells $/ 10 \mathrm{ml}$ in $10 \mathrm{~cm}$ dishes and incubated for $24 \mathrm{~h}$. Then, the sample ( $5 \mu$ l of ethanolic solution) was added to the medium to achieve the desired final concentration of $10 \mu \mathrm{M}-\mathrm{CUR},-\mathrm{THC}$ or -CURG. The final concentration of ethanol in the medium was $0.05 \%(\mathrm{v} / \mathrm{v})$, which did not affect cell viability. After incubation for $10 \mathrm{~min}, 2 \mathrm{~h}$ and $24 \mathrm{~h}$, the cells were counted using a haemocytometer. Curcuminoids were extracted from the cells (or medium) and analysed by HPLC as described below.

THP-1 macrophages were prepared from THP-1 monocytes $\left(2 \times 10^{6}\right.$ cells $)$ by incubating them with $100 \mathrm{~nm}$-phorbol 12-myristate 13-acetate (PMA) for $24 \mathrm{~h}$. THP-1 macrophages were then treated with $10 \mu \mathrm{m}-\mathrm{CUR}$, -THC or -CURG for $10 \mathrm{~min}, 2 \mathrm{~h}$ and $24 \mathrm{~h}$. After extraction, the samples were subjected to HPLC analysis.

\section{HPLC analysis}

Based on our previous method ${ }^{(11)}$, cellular and medium curcuminoids were analysed by HPLC. Briefly, the cells $\left(0 \cdot 5-3 \times 10^{6}\right)$ were subjected to sonication with $200 \mu \mathrm{l}$ of water. The solution was mixed with $400 \mu$ l of methanol by vortexing and centrifuged at $5000 \mathrm{~g}$ for $15 \mathrm{~min}$ at $4^{\circ} \mathrm{C}$. The supernatant was collected, mixed with $1 \mathrm{ml}$ of water and loaded onto an Oasis HLB 1 cc cartridge (Waters). The cartridge was washed with $1 \mathrm{ml}$ of water, and curcuminoids were eluted with $2 \mathrm{ml}$ of methanol. Culture medium $(2 \mathrm{ml})$ was directly loaded onto the Oasis HLB $1 \mathrm{cc}$ cartridge. The cartridge was washed with $1 \mathrm{ml}$ of water and eluted with $2 \mathrm{ml}$ of methanol.

Aliquots of cell extracts were injected onto a C18 column (Nova-Pak C18, $3.9 \times 150 \mathrm{~mm}$; Waters) kept at $40^{\circ} \mathrm{C}$. The mobile phase consisted of two components: A, $0.05 \%$ formic acid ( $\mathrm{pH} 3 \cdot 0$ ), and $\mathrm{B}$, acetonitrile. The gradient profile was as follows: $0-30 \mathrm{~min}, 85-0 \%$ A linear. The flow rate was $0.5 \mathrm{ml} / \mathrm{min}$. The fluorescence (FL) of curcuminoids was detected at an excitation wavelength of $426 \mathrm{~nm}$ and an emission wavelength of $539 \mathrm{~nm}$ and UV absorbance of curcuminoids was also detected at $280 \mathrm{~nm}$ and $420 \mathrm{~nm}$.

In the detailed analysis of CUR metabolites, aliquots of cell (or medium) extracts were injected onto a C18 column (XBridge C18, $2.1 \times 150 \mathrm{~mm}$; Waters) kept at $40^{\circ} \mathrm{C}$. The mobile phase consisted of two components: A, 0.05\% formic acid ( $\mathrm{pH} \mathrm{3.0)}$, and $\mathrm{B}$, acetonitrile. The gradient profile was as follows: $0-30 \mathrm{~min}, 85-0 \% \mathrm{~A}$ linear. The flow rate was $0.2 \mathrm{ml} / \mathrm{min}$. Curcuminoids were analysed using API 3200 QTRAP HPLC-MS/MS (AB Sciex). MS/MS parameters were adjusted with CUR, CURG and THC standards under electrospray ionisation (negative). The parameters were as follows: collision energy, $-60 \mathrm{~V}$; declustering potential, $-45 \mathrm{~V}$; turbo gas temperature, $300^{\circ} \mathrm{C}$; spray voltage, $-4500 \mathrm{~V}$; nebuliser gas, 30 psi; auxiliary gas, 30 psi; curtain gas, 20 psi; collision gas, medium. CUR, CURG and THC were detected using multiple-reaction monitoring (MRM) for the transition of parent ions to product ions: CUR, $\mathrm{m} / z 367>134$; CURG, $\mathrm{m} / z$ $543>134$; THC, $m / z 371>135$. The concentrations of cellular and medium CUR, THC and CURG were calculated using the standard curves of CUR, THC and CURG (online supplementary Fig. S1). Other curcuminoids were analysed using literature-known MRM transitions ${ }^{(17,18)}$ : curcumin sulphate (CURS), $m / z 447>134$; curcumin glucuronide sulphate (CURGS), $m / z 623>134$; curcumin diglucuronide (CURDG), $m / z \quad 719>134 ; \quad$ curcumin disulphate (CURDS), $\mathrm{m} / \mathrm{z}$ $527>134$; dihydrocurcumin (DHC), $m / z 369>135$; hexahydrocurcumin (HHC), $m / z 373>179$; octahydrocurcumin (OHC), $m / z 375>179$; dihydrocurcumin glucuronide (DHCG), $m / z \quad 545>135$; tetrahydrocurcumin glucuronide (THCG), $m / z 547>135$; hexahydrocurcumin glucuronide (HHCG), $m / z$ $549>179$; octahydrocurcumin glucuronide (OHCG), $m / z$ $551>179$; dihydrocurcumin sulphate (DHCS), $m / z 449>135$; tetrahydrocurcumin sulphate (THCS), $m / z \quad 451>135$; hexahydrocurcumin sulphate (HHCS), $m / z 453>179$; octahydrocurcumin sulphate (OHCS), $m / z 455>179$.

\section{Effect of curcuminoids on cellular lipid update}

To investigate the effect of curcuminoids on lipid accumulation, THP-1 macrophages were prepared from THP-1 monocytes $\left(1 \times 10^{6}\right.$ cells $/ 3 \mathrm{ml}$ in six-well plates $)$ by incubating monocytes with $100 \mathrm{~nm}$-PMA for $48 \mathrm{~h}$. The cells were then treated with or without $10 \mu \mathrm{m}$-CUR, -THC or -CURG for $24 \mathrm{~h}$, and cellular lipids were identified by Oil Red $O$ staining and by measuring the extracted red-stained neutral lipids using a spectrometer set at $490 \mathrm{~nm}^{(19)}$. The effect of $10 \mu \mathrm{m}$-demethoxycurcumin (DMC; Sigma) and bisdemethoxycurcumin (BDMC; Sigma) on cellular lipid uptake was determined.

\section{Evaluation of evidence for a curcumin transporter}

As CUR, DMC and BDMC share similar molecular structures, their cellular uptake was investigated to evaluate a possibility for the presence of a CUR transporter. CUR reagent of a crude grade was obtained from Sigma. The crude CUR consists of approximately $72 \%$ CUR, 17\% DMC and 10\% BDMC. THP-1 monocytes were seeded at $1 \times 10^{6}$ cells $/ 3 \mathrm{ml}$ in six-well 
(a)
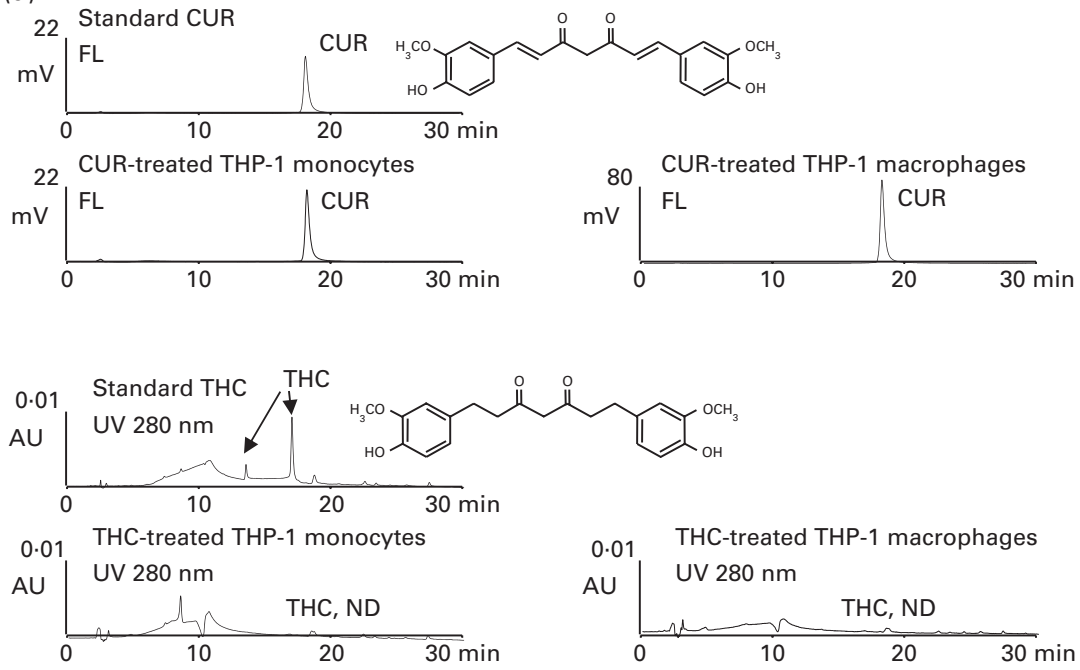

(b)
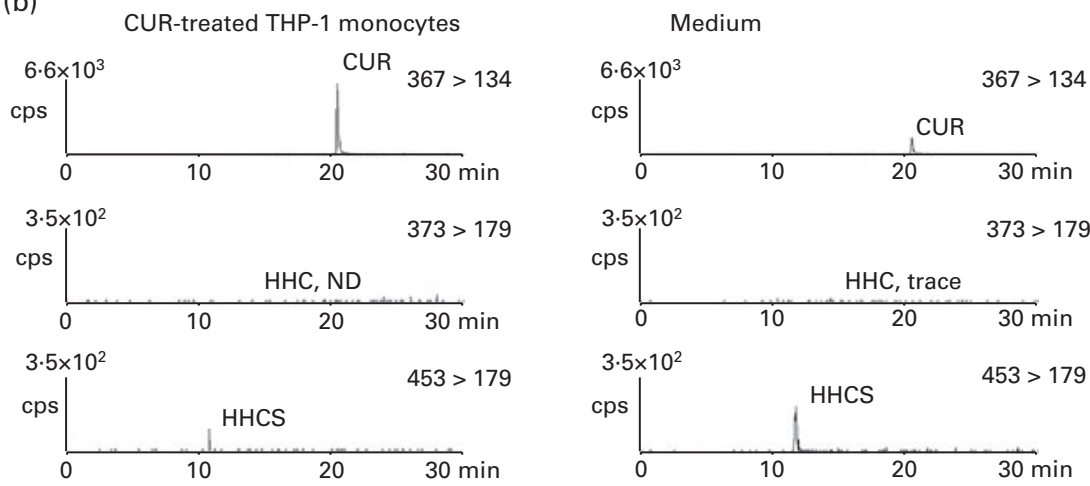

THC-treated THP-1 monocytes
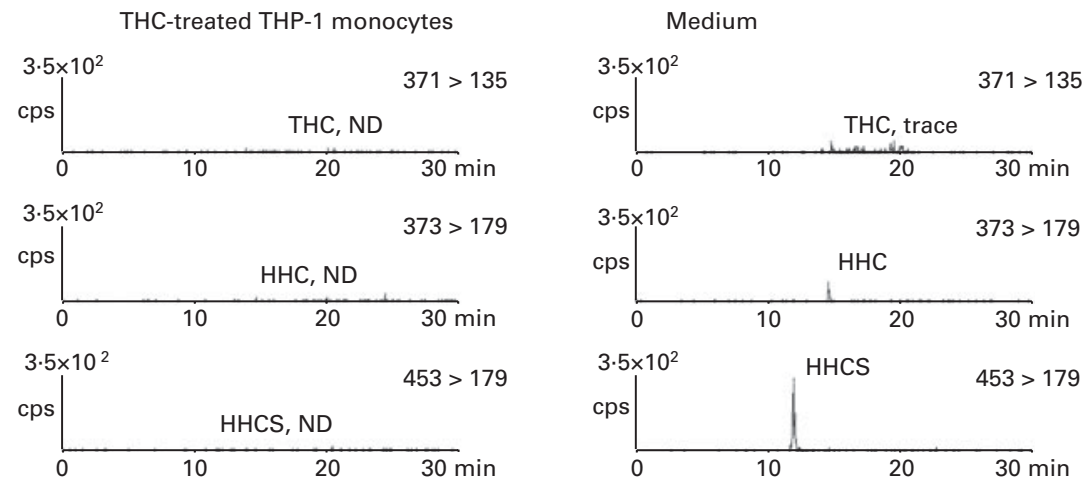

Fig. 1. Conventional HPLC (a) and HPLC-MS/MS (b) analyses of the cellular uptake and metabolism of curcuminoids. (a) Standard curcumin (CUR; $0.03 \mathrm{nmol}$ ) was analysed by HPLC-fluorescence (FL). THP-1 monocytes or THP-1 macrophages (induced to differentiate with 100 nM-phorbol 12-myristate 13-acetate for $24 \mathrm{~h}$ ) were treated with $10 \mu \mathrm{M}$-CUR for $24 \mathrm{~h}$, and the cell extracts were subjected to HPLC-FL analysis. Standard tetrahydrocurcumin (THC; $0.1 \mathrm{nmol}$ ) and extracts from cells treated with THC for $24 \mathrm{~h}$ were analysed by HPLC-UV at $280 \mathrm{~nm}$. Standard THC exhibited a double peak, as a rapid transition would occur between the keto-enol $\beta$-diketone molecular species of $\mathrm{THC}^{(27)}$. A somewhat unstable baseline was observed due to gradient analysis close to the detection limit. (b) THP1 monocytes were treated with $10 \mu \mathrm{M}$-CUR or $-\mathrm{THC}$ for $24 \mathrm{~h}$, and the cell and medium extracts were analysed by HPLC-MS/MS with multiple-reaction monitoring. Chromatograms of representative curcuminoids (CUR, THC, hexahydrocurcumin (HHC) and hexahydrocurcumin sulphate (HHCS)) are shown. Other curcuminoids (curcumin glucuronide, $543>134$; curcumin sulphate, $447>134$; curcumin glucuronide sulphate, $623>134$; curcumin diglucuronide, $719>134$; curcumin disulphate, $527>134$; dihydrocurcumin, $369>135$; octahydrocurcumin, $375>179$; dihydrocurcumin glucuronide, $545>135$; tetrahydrocurcumin glucuronide, $547>135$; hexahydrocurcumin glucuronide, $549>179$; octahydrocurcumin glucuronide, $551>179$; dihydrocurcumin sulphate, $449>135$; tetrahydrocurcumin sulphate, $451>135$; and octahydrocurcumin sulphate, $455>179$ ) were not detected (ND) or traces were detected. For all HPLC analyses, cell extracts (corresponding to $0.05-1.5 \times 10^{4}$ cells) or medium extracts (corresponding to $2-10 \mu l$ medium) were analysed by HPLC-FL, HPLC-UV or MS/MS analysis. Each chromatogram is representative of duplicate HPLC analysis from independently treated samples. Detailed analytical conditions are described in the Materials and methods section. AU, arbitrary units; cps, centipoise. 
plates and incubated for $24 \mathrm{~h}$. The cells were then treated with $10 \mu \mathrm{M}$-crude CUR for $2 \mathrm{~h}$. Cellular curcuminoids were analysed by HPLC-FL as described above.

\section{Statistical analysis}

Data are expressed as means with their standard errors. When more than two groups were compared, ANOVA was carried out, followed by Bonferroni post hoc test. Differences were considered to be significant at $P<0.05$.

\section{Results and discussion}

\section{Conventional HPLC analysis of the cellular uptake and metabolism of curcuminoids}

In our previous in vitro study ${ }^{(6)}$, we treated THP-1 monocytes and macrophages (induced to differentiate with $100 \mathrm{~nm}$-PMA for $24 \mathrm{~h}$ ) with $10 \mu \mathrm{m}$-CUR for $24 \mathrm{~h}$ and found a marked accumulation of lipids in the cells, but no such effect was observed when THC, CUR metabolite, was tested. To further understand these differential effects, cellular CUR and THC concentrations were first evaluated by conventional HPLC-FL and HPLC-UV analyses, respectively, under the same conditions (Fig. 1(a)).

With reference to the cellular uptake of CUR, Jaruga et al. ${ }^{(20)}$ reported that CUR readily penetrates into the cytoplasm of rat thymocytes and accumulates in membranous structures including the endoplasmic reticulum. Subsequently, Dempe et al. ${ }^{(14,16)}$ reported that when Caco-2 and HepG2 cells are treated with CUR, CUR is rapidly taken up (possibly within minutes) by these cells and transiently accumulated in the endoplasmic reticulum. Most of the CUR then undergoes a stepwise reduction of olefinic double bonds, leading to
THC, HHC and OHC in these cells. Both CUR and reductive metabolites are further conjugated with glucuronic acid and sulphate in Caco-2 cells. Thus, with longer incubation time (e.g. 6-30 h), unmetabolised CUR remains at very low concentrations in Caco-2 and HepG2 cells. However, in the present study, after incubation of THP-1 monocytes/ macrophages with CUR for $24 \mathrm{~h}$, the cell lysates still exhibited the yellow colour of CUR (data not shown); thus, a large peak of CUR was detectable on the FL chromatograms of the cell extracts (Fig. 1(a)). On the other hand, when THP-1 monocytes/macrophages were incubated with $10 \mu \mathrm{m}$-THC for $24 \mathrm{~h}$, there were no detectable levels of THC on cell chromatograms. These results suggest that in THP-1 monocytes/macrophages, CUR and THC are taken up and metabolised differently, further suggesting a possible explanation for the previously observed differences in the effects of CUR and THC on lipid accumulation in THP-1 monocytes/ macrophages ${ }^{(6)}$.

\section{HPLC-MS/MS analysis of the cellular uptake and metabolism of curcuminoids}

To evaluate the cellular uptake and metabolism of curcuminoids further, we analysed CUR- and THC-treated THP-1 monocytes/macrophages by HPLC-MS/MS. Considering the limitations of conventional HPLC-FL and HPLC-UV methods for detailed analysis of various metabolites, HPLC-MS/MS analysis has specific advantages over the conventional analysis of biomolecules. Furthermore, HPLC-MS/MS analysis provides neutral loss scanning and MRM, which in turn provide useful structural information of the analytes even in the presence of background contaminants in complex biological systems $^{(21)}$. We and other researchers have examined and identified several CUR metabolites from the plasma and tissues

Table 1. Time course of changes in curcuminoid levels when THP-1 monocytes/macrophages were treated with $10 \mu \mathrm{M}$-curcumin (CUR), -tetrahydrocurcumin (THC) or -curcumin glucuronide (CURG)*

\begin{tabular}{|c|c|c|c|c|c|c|c|c|c|c|c|}
\hline Treatments & Time & CUR & THC & CURG & HHCS† & Treatment & Time & CUR & THC & CURG & HHCS† \\
\hline \multicolumn{6}{|c|}{ THP-1 monocytes $\left(\mathrm{pmol} / 2.0 \times 10^{6} \mathrm{cells}\right)$} & \multicolumn{6}{|c|}{ THP-1 monocytes $\left(\mathrm{pmol} / 2.0 \times 10^{6}\right.$ cells $)$} \\
\hline \multirow[t]{3}{*}{ CUR } & $10 \min$ & 1313 & 0 & 0 & 0 & \multirow[t]{3}{*}{ CUR } & $10 \min$ & 2073 & 0 & 0 & 0 \\
\hline & $2 \mathrm{~h}$ & 2029 & 0 & 0 & 0 & & $2 \mathrm{~h}$ & 2737 & 0 & 0 & 0 \\
\hline & $24 \mathrm{~h}$ & 401 & 0 & 0 & 86 & & $24 \mathrm{~h}$ & 459 & 0 & 0 & 0 \\
\hline \multirow[t]{3}{*}{ THC } & $10 \mathrm{~min}$ & - & 0 & - & 0 & \multirow[t]{3}{*}{ THC } & $10 \mathrm{~min}$ & - & 0 & - & 0 \\
\hline & $2 \mathrm{~h}$ & - & 0 & - & 0 & & $2 \mathrm{~h}$ & - & 0 & - & 0 \\
\hline & $24 \mathrm{~h}$ & - & 0 & - & 0 & & $24 \mathrm{~h}$ & - & 0 & - & 0 \\
\hline \multirow[t]{3}{*}{ CURG } & $10 \mathrm{~min}$ & 0 & 0 & 0 & - & \multirow[t]{3}{*}{ CURG } & $10 \mathrm{~min}$ & 0 & 0 & 5 & - \\
\hline & $2 \mathrm{~h}$ & 0 & 0 & 19 & - & & $2 \mathrm{~h}$ & 0 & 0 & 2 & - \\
\hline & $24 \mathrm{~h}$ & 0 & 0 & 0 & - & & $24 \mathrm{~h}$ & 0 & 0 & 2 & - \\
\hline \multicolumn{6}{|c|}{ Medium $(\mu \mathrm{M})$} & \multicolumn{6}{|c|}{ Medium $(\mu \mathrm{M})$} \\
\hline \multirow[t]{3}{*}{ CUR } & $10 \mathrm{~min}$ & $4 \cdot 1$ & 0 & 0 & 0 & \multirow[t]{3}{*}{ CUR } & $10 \mathrm{~min}$ & $6 \cdot 4$ & 0 & 0 & 0 \\
\hline & $2 \mathrm{~h}$ & 0.7 & Trace & 0 & 0 & & $2 \mathrm{~h}$ & $2 \cdot 1$ & Trace & 0 & 0 \\
\hline & $24 \mathrm{~h}$ & 0.1 & 0 & 0 & 2592 & & $24 \mathrm{~h}$ & 0.3 & 0 & 0 & 4839 \\
\hline \multirow[t]{3}{*}{ THC } & $10 \mathrm{~min}$ & - & $8 \cdot 3$ & - & 0 & \multirow[t]{3}{*}{ THC } & $10 \mathrm{~min}$ & - & $9 \cdot 9$ & - & 0 \\
\hline & $2 \mathrm{~h}$ & - & 1.9 & - & 0 & & $2 \mathrm{~h}$ & - & $3 \cdot 1$ & - & 0 \\
\hline & $24 \mathrm{~h}$ & - & Trace & - & 4906 & & $24 \mathrm{~h}$ & - & Trace & - & 4150 \\
\hline \multirow[t]{3}{*}{ CURG } & $10 \mathrm{~min}$ & 0 & 0 & 6.9 & - & \multirow[t]{3}{*}{ CURG } & $10 \mathrm{~min}$ & 0 & 0 & 9.2 & - \\
\hline & $2 \mathrm{~h}$ & 0 & 0 & 4.8 & - & & $2 \mathrm{~h}$ & 0 & 0 & 1.3 & - \\
\hline & $24 \mathrm{~h}$ & 0 & 0 & $1 \cdot 1$ & - & & $24 \mathrm{~h}$ & 0 & 0 & 1.3 & - \\
\hline
\end{tabular}

HHCS, hexahydrocurcumin sulphate.

*Values are represented as the mean of duplicate HPLC-MS/MS analysis of independently treated samples.

†Peak areas (counts/injection) of HHCS are shown due to unavailability of a standard and difficulty in determination. 
of rats or mice supplemented with CUR using HPLC-MS/MS analysis with MRM $^{(11,17,18,22,23)}$. HPLC-MS/MS analysis of curcuminoids in liver microsomes ${ }^{(24)}$ and in turmeric ${ }^{(25)}$ has also been reported. In the present study, we used this method to determine the number of curcuminoids that might possibly be present in the cells or in the cell-culture medium, such as CUR, CURG, CURS, CURGS, CURDG, CURDS, DHC, THC, HHC, OHC, DHCG, THCG, HHCG, OHCG, DHCS, THCS, HHCS and OHCS (Fig. 1(b)). The detection of these curcuminoids by HPLC-MS/MS analysis was reproducible and was not altered by the storage of cellular and medium extract samples at below $-30^{\circ} \mathrm{C}$ for 1 month (data not shown).

In agreement with the results of conventional HPLC analysis (Fig. 1(a)), a considerable amount of CUR $(\mathrm{m} / \mathrm{z} 367>134)$ was detected in MRM chromatograms after incubation of THP-1 monocytes with $10 \mu \mathrm{m}$-CUR for $24 \mathrm{~h}$ (Fig. 1(b)). There were no detectable levels of THC in THP-1 monocytes treated with $10 \mu \mathrm{m}$-THC for $24 \mathrm{~h}$. Similar chromatograms were obtained from the samples of THP-1 macrophages (data not shown). Interestingly, in both CUR and THC treatments, metabolites such as HHC $(\mathrm{m} / z 373>179)$ and HHCS $(\mathrm{m} / z$ $453>179)$ could be identified, especially in the cell-culture medium, and HHCS was found to be the predominant metabolite. Based on these results (Fig. 1) and those of the time-course data (Table 1), the following conclusions were drawn: (1) CUR is readily taken up by THP-1 monocytes/ macrophages and metabolised mainly to HHCS; (2) metabolites are released into the cell-culture medium; (3) a significant amount of CUR still remains in the cells even after $24 \mathrm{~h}$, suggesting that the metabolic modifications of CUR (e.g. conversion of CUR to DHC) are slower than its uptake; (4) unlike CUR, THC is taken up more slowly and is more rapidly converted to secondary metabolites (e.g. HHCS), resulting in no detectable levels of THC in THP-1 monocytes/macrophages; (5) interestingly, THP-1 cells are devoid of glucuronidation of curcuminoids. These possible events are summarised in Fig. 2. Based on these data, it is therefore likely that in our previous in vitro study ${ }^{(6)}$, the active compound responsible for the lipid-accumulating effect in THP-1 monocytes/macrophages would be CUR itself. The lack of an effect of THC might be mainly due to its low absorption and rapid elimination from the cells.

\section{Effects of curcuminoids on lipid uptake by THP-1 monocytes/macrophages}

Ireson et $a .^{(26)}$ tested the effects of CUR and metabolites (CURS, THC, HHC and OHC) on the inhibition of

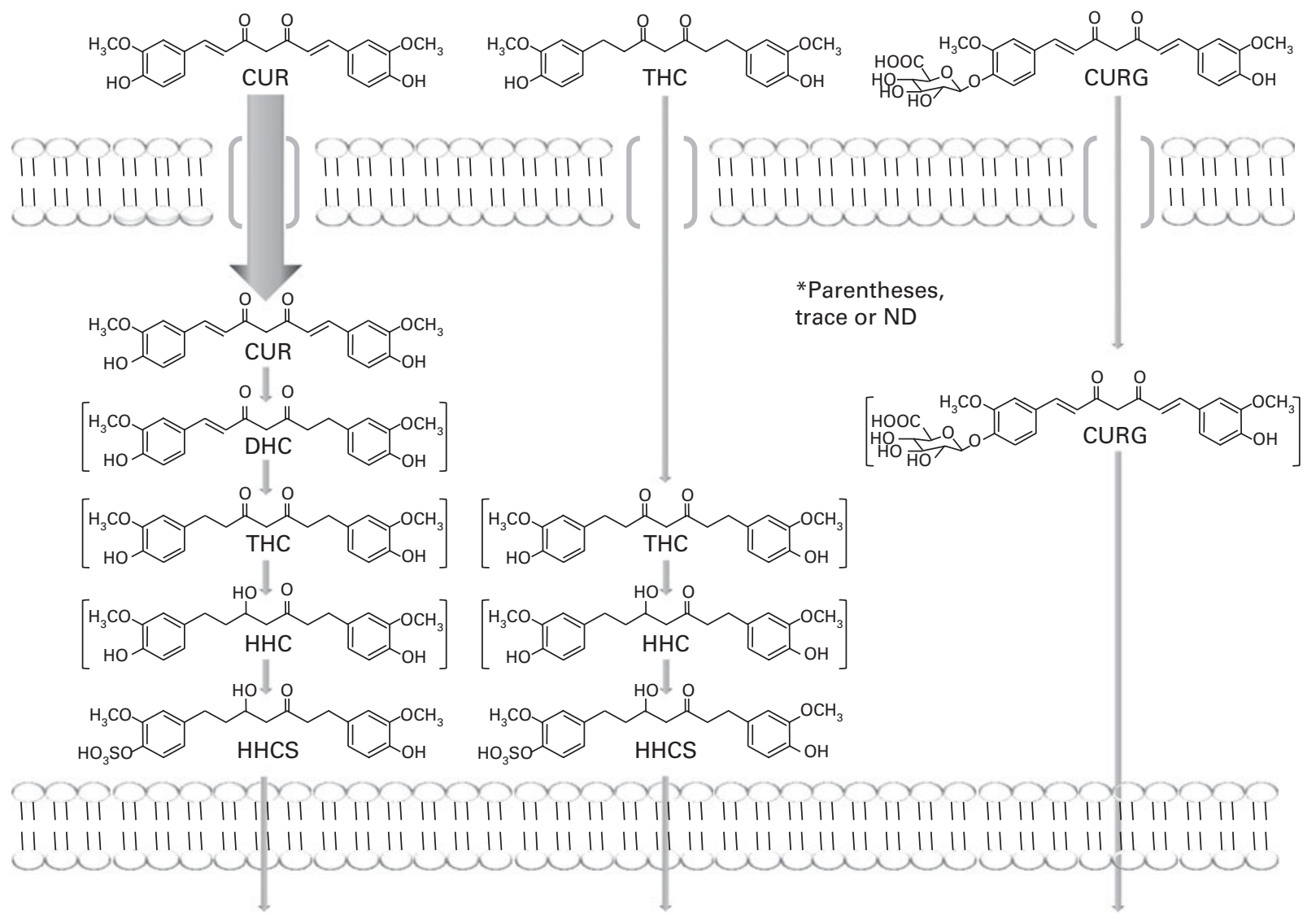

Fig. 2. Proposed cellular uptake and metabolism of curcumin (CUR), tetrahydrocurcumin (THC) and curcumin glucuronide (CURG) in THP-1 monocytes/macrophages. CUR is rapidly taken up by THP-1 cells and remains in the cells for longer incubation periods (e.g. $24 \mathrm{~h}$ ), probably due to the slow conversion of CUR to its metabolite (dihydrocurcumin (DHC)). Unlike that of CUR, the cellular uptake of THC is low and slow. Even though incorporated, THC readily undergoes reduction and sulphation to mainly hexahydrocurcumin sulphate (HHCS). Once metabolised, metabolites (e.g. HHCS) are quickly recovered in the medium. Similar to THC, CURG is hardly incorporated into THP-1 cells. HHC, hexahydrocurcumin. 
PMA-induced $\mathrm{PGE}_{2}$ production in human colonic epithelial cells. They found that the bioactivity of these metabolites was weaker than that of unmetabolised CUR. Considering our findings (Fig. 1 and Table 1) and those reported by Ireson et $a l .{ }^{(26)}$, it is possible to deduce that the cellular metabolism of curcuminoids determines their biological activity. Based on the fact that curcuminoids are present primarily as CURG in the body rather than as CUR or other metabolites $^{(9-11)}$, we thought that it would be most important to determine the metabolism and bioactivity of CURG, which have not been thoroughly investigated. In the present study, when THP-1 monocytes/macrophages were incubated with $10 \mu \mathrm{m}$-CURG, very low levels of CURG were found in the cells (Table 1). Other metabolites were not detectable in the $10 \mu \mathrm{M}$-CURG-treated THP-1 monocytes/macrophages. These findings suggest that polar metabolites such as CURG are hardly taken up and metabolised by THP-1 cells, which may explain why CURG did not induce lipid accumulation in THP-1 macrophages (Fig. 3(a)). By contrast, in our earlier study $^{(6)}$ and the present study (Fig. 3(a)), we found that CUR increased lipid accumulation in THP-1 macrophages. As has been pointed out above, CUR-induced accumulation of lipids in macrophages may be part of a mechanism aimed at the removal of lipids from the bloodstream, but in contrast to the results of in vitro studies, those of our in vivo studies have indicated a trend towards reduction of lipid levels in peritoneal macrophages in LDL receptor knockout mice fed a

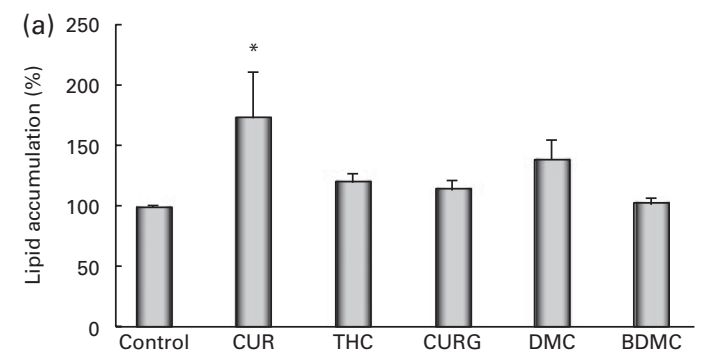

(b)
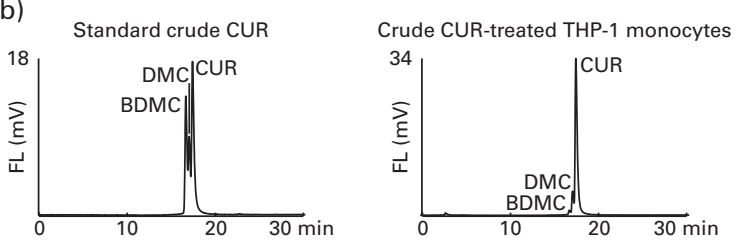

Fig. 3. Effects of curcuminoids on cellular lipid levels in THP-1 cells (a) and differential cellular uptake of curcuminoids into cells (b). (a) THP-1 macrophages (induced to differentiate with $100 \mathrm{~nm}$-phorbol 12-myristate 13-acetate for $48 \mathrm{~h}$ ) were treated with or without $10 \mu \mathrm{M}$-curcumin (CUR), -tetrahydrocurcumin (THC), -curcumin glucuronide (CURG), -demethoxycurcumin (DMC) or -bisdemethoxycurcumin (BDMC) for $24 \mathrm{~h}$. Lipid accumulation was measured by Oil Red $O$ staining. Values are means with their standard errors represented by vertical bars $(n 9)$. (b) Differential cellular uptake of CUR, DMC and BDMC in THP-1 cells. Standard crude CUR (corresponding to $0.03 \mathrm{nmol}$ CUR) and extracts from THP-1 monocytes treated with crude CUR for $2 \mathrm{~h}$ were analysed by HPLC-fluorescence (FL). CUR, DMC and BDMC were all clearly detected in the analysis of standard crude CUR, whereas a predominant CUR peak was observed in the analysis of the extract of crude CURtreated THP-1 monocytes. Each chromatogram is representative of duplicate HPLC analysis. Detailed analytical conditions are described in the Materials and methods section. high-fat diet for 4 months and supplemented with $\mathrm{CUR}^{(6)}$ From the present results and our earlier findings, one may deduce the following for the differential effects of CUR in vitro $v$. in vivo: in the in vitro system, CUR is the stimulator of lipid uptake by macrophages, whereas in the in vivo system, the majority of CUR is metabolised and present as metabolites in the plasma (e.g. CURG) that are unable to up-regulate lipid accumulation in circulating monocytes/ macrophages. Moreover, it is plausible that the metabolites of CUR may regulate the expression of lipid metabolism genes leading to a lower accumulation of lipids in macrophage or adipocytes in vivo ${ }^{(5)}$. In the liver, CUR may affect the uptake/metabolism/excretion of fatty acids before CUR is metabolised, leading to a reduction of overall plasma lipid levels ${ }^{(5)}$. It remains to be determined whether such a hypolipidaemic effect induced by CUR contributes to the reduction of lipid accumulation observed in peritoneal macrophages. Further in vitro and in vivo studies are needed to better understand the regulatory effects of CUR, CURG and other metabolites including THC on lipid accumulation in the body.

On the other hand, in the present study, a time-dependent decrease of curcuminoid (CUR, THC, and CRUG) levels was found in the culture medium (Table 1). This may be partly explained by the low stability of the curcuminoids in the medium, as curcuminoids such as CUR are said to be gradually decomposed in the medium without cells ${ }^{(14)}$

\section{Possible curcumin transporter in the cells}

In addition to the above-mentioned observations, we found distinct differences in the cellular uptake of CUR, THC and CURG (Table 1). Similar results were obtained in HepG2 cells $^{(11)}$ and other cells such as human intestinal epithelial Caco-2 cells, human embryonic kidney 293 (HEK293) cells and mouse RAW264.7 macrophages (data not shown). The data showing a different uptake of CUR, relative to the other metabolites, may imply the existence of a CUR transporter in these cells, which is described herein for the first time. Although CUR, DMC and BDMC share very similar molecular structures, the cellular uptake of lipids was found exclusively in the CUR-treated THP-1 cells (Fig. 3(a)). Thus, CUR-induced lipid uptake in THP-1 cells is an event that may be related to CUR transport inside the cells. As anticipated, when THP-1 monocytes were treated with crude CUR (containing CUR, DMC and BDMC) for $2 \mathrm{~h}$, CUR was preferentially incorporated into the cells (Fig. 3(b)). Similar results were obtained at other incubation time points (e.g. 1 and $3 \mathrm{~h}$ ) (data not shown). Therefore, the transporter may be able to recognise and transport CUR quite selectively into the cells, though we do not provide direct experimental evidence for the presence of CUR transporter(s). Despite the fact that the existence of a CUR transporter appears to be the best explanation for the transport specificity, other possibilities (e.g. simple diffusion of curcuminoids into cells due to their different polarities and/or solubilities) cannot be ruled out. Obtaining direct evidence of a CUR transporter is part of ongoing studies, involving saturation kinetic analyses, inhibitor usage and temperature dependence of the phenomenon. 


\section{Supplementary material}

To view supplementary material for this article, please visit http://dx.doi.org/10.1017/S0007114514000567

\section{Acknowledgements}

The authors thank Stephanie Marco for her assistance in the preparation of the manuscript.

The present study was supported in part by the USDA/ARS under contract no. 58-1950-0-014 and sabbatical fellowship from Tohoku University (Japan) to K. N. Any opinions, findings, conclusions or recommendations expressed in this article are those of the author(s) and do not necessarily reflect the view of the US Department of Agriculture.

The authors' contributions are as follows: K. N. and J.-M. Z. were involved in data collection, data analysis, data interpretation, literature search and manuscript preparation; S. H. K., M. J. T. and G. G. D. were involved in data collection, data analysis and data interpretation; A. A., T. M. and M. M. were involved in study design, data interpretation and manuscript review. All authors read and approved the final manuscript.

None of the authors has any conflicts of interest to declare.

\section{References}

1. Gupta SC, Sung B, Kim JH, et al. (2013) Multitargeting by turmeric, the golden spice: from kitchen to clinic. Mol Nutr Food Res 57, 1510-1528.

2. Asai A \& Miyazawa T (2001) Dietary curcuminoids prevent high-fat diet-induced lipid accumulation in rat liver and epididymal adipose tissue. J Nutr 131, 2932-2935.

3. Sharma RA, Gescher AJ \& Steward WP (2005) Curcumin: the story so far. Eur J Cancer 41, 1955-1968.

4. Ejaz A, Wu D, Kwan P, et al. (2009) Curcumin inhibits adipogenesis in 3T3-L1 adipocytes and angiogenesis and obesity in C57/BL mice. J Nutr 139, 919-925.

5. Zingg JM, Hasan ST \& Meydani M (2013) Molecular mechanisms of hypolipidemic effects of curcumin. Biofactors 39, $101-121$

6. Zingg JM, Hasan ST, Cowan D, et al. (2012) Regulatory effects of curcumin on lipid accumulation in monocytes/ macrophages. J Cell Biochem 113, 833-840.

7. Sawada H, Saito Y \& Noguchi N (2012) Enhanced CD36 expression changes the role of $N r f 2$ activation from antiatherogenic to pro-atherogenic in apoE-deficient mice. Atherosclerosis 225, 83-90.

8. Kou MC, Chiou SY, Weng CY, et al. (2013) Curcuminoids distinctly exhibit antioxidant activities and regulate expression of scavenger receptors and heme oxygenase-1. Mol Nutr Food Res 57, 1598-1610.

9. Asai A \& Miyazawa T (2000) Occurrence of orally administered curcuminoid as glucuronide and glucuronide/sulfate conjugates in rat plasma. Life Sci 67, 2785-2793.

10. Anand P, Kunnumakkara AB, Newman RA, et al. (2007) Bioavailability of curcumin: problems and promises. $\mathrm{Mol}$ Pharm 4, 807-818.

11. Shoji M, Nakagawa K, Watanabe A, et al. (2014) Comparison of the effects of curcumin and curcumin glucuronide in human hepatocellular carcinoma HepG2 cells. Food Chem 151, 126-132.
12. Pan MH, Huang TM, Lin JK, et al. (1999) Biotransformation of curcumin through reduction and glucuronidation in mice. Drug Metab Dispos 27, 486-494.

13. Usta M, Wortelboer HM, Vervoort J, et al. (2007) Human glutathione $S$-transferase-mediated glutathione conjugation of curcumin and efflux of these conjugates in Caco-2 cells. Chem Res Toxicol 20, 1895-1902.

14. Dempe JS, Pfeiffer E, Grimm AS, et al. (2008) Metabolism of curcumin and induction of mitotic catastrophe in human cancer cells. Mol Nutr Food Res 52, 1074-1081.

15. Kumar A, Li L, Chaturvedi A, et al. (2012) Two-photon fluorescence properties of curcumin as a biocompatible marker for confocal imaging. Appl Phys Lett 100, 203701.

16. Dempe JS, Scheerle RK, Pfeiffer E, et al. (2013) Metabolism and permeability of curcumin in cultured Caco-2 cells. Mol Nutr Food Res 57, 1543-1549.

17. Marczylo TH, Verschoyle RD, Cooke DN, et al. (2007) Comparison of systemic availability of curcumin with that of curcumin formulated with phosphatidylcholine. Cancer Chemother Pharmacol 60, 171-177.

18. Marczylo TH, Steward WP \& Gescher AJ (2009) Rapid analysis of curcumin and curcumin metabolites in rat biomatrices using a novel ultraperformance liquid chromatography (UPLC) method. J Agric Food Chem 57, 797-803.

19. Ramírez-Zacarías JL, Castro-Muñozledo F \& Kuri-Harcuch W (1992) Quantitation of adipose conversion and triglycerides by staining intracytoplasmic lipids with Oil red O. Histochemistry 97, 493-497.

20. Jaruga E, Salvioli S, Dobrucki J, et al. (1998) Apoptosis-like, reversible changes in plasma membrane asymmetry and permeability, and transient modifications in mitochondrial membrane potential induced by curcumin in rat thymocytes. FEBS Lett 433, 287-293.

21. King R \& Fernandez-Metzler C (2006) The use of Qtrap technology in drug metabolism. Curr Drug Metab 7, 541-545.

22. Zhongfa L, Chiu M, Wang J, et al. (2012) Enhancement of curcumin oral absorption and pharmacokinetics of curcuminoids and curcumin metabolites in mice. Cancer Chemother Pharmacol 69, 679-689.

23. Verma MK, Najar IA, Tikoo MK, et al. (2013) Development of a validated UPLC-qTOF-MS method for the determination of curcuminoids and their pharmacokinetic study in mice. Daru 21, 11.

24. Tamvakopoulos C, Sofianos ZD, Garbis SD, et al. (2007) Analysis of the in vitro metabolites of diferuloylmethane (curcumin) by liquid chromatography-tandem mass spectrometry on a hybrid quadrupole linear ion trap system: newly identified metabolites. Eur J Drug Metab Pharmacokinet 32, 51-57.

25. Jiang H, Timmermann BN \& Gang DR (2006) Use of liquid chromatography-electrospray ionization tandem mass spectrometry to identify diarylheptanoids in turmeric (Curcuma longa L.) rhizome. J Chromatogr A 1111, 21-31.

26. Ireson C, Orr S, Jones DJ, et al. (2001) Characterization of metabolites of the chemopreventive agent curcumin in human and rat hepatocytes and in the rat in vivo, and evaluation of their ability to inhibit phorbol ester-induced prostaglandin $\mathrm{E}_{2}$ production. Cancer Res 61, 1058-1064.

27. Liu A, Lou H, Zhao L, et al. (2006) Validated LC/MS/MS assay for curcumin and tetrahydrocurcumin in rat plasma and application to pharmacokinetic study of phospholipid complex of curcumin. J Pharm Biomed Anal 40, 720-727. 